\title{
Tendencias de los accidentes de tránsito en Ecuador: 2000-2015*
}

\section{Trends in Traffic Accidents in Ecuador: 2000-2015}

\section{Tendências em acidentes de trânsito no Equador: 2000-2015}

Fecha de recepción: 23-01-2017 Fecha de aceptación: 22-03-2017 Disponible en línea: 30-05-2017 doi:10.11144/Javeriana.rgps16-33.tate

Cómo citar este artículo:

Algora-Buenafé AF, Russo-Puga M, Suasnavas-Bermúdez PR, Merino-Salazar P, Gómez-García AR. Tendencias de los accidentes de tránsito en Ecuador: 2000-2015. Rev Gerenc Polít Salud. 2017; 16 (33): 52-58. https://doi.org/10.11144/Javeriana.rgps16-33.tate

\author{
Alfonso Francisco Algora-Buenafé** \\ Marcel Russo-Puga ${ }^{* * *}$ \\ Pablo Roberto Suasnavas-Bermúdez ${ }^{* * * *}$ \\ Pamela Merino-Salazar ${ }^{* * * * *}$ \\ Antonio Ramón Gómez-García*****
}

* Artículo de investigación. Por favor, remitir la correspondencia a Antonio Ramón Gómez García, Universidad Internacional SEK, Campus Miguel de Cervantes, Alberto Einstein, s/n y 5 ta transversal - Carcelén. Quito, Ecuador. Tel: (593 2) 3974800. Ext.: 169. Correo electrónico: antonio.gomez@uisek.edu.ec

** Ph. D. en Educación, profesor titular de la Facultad de Ciencias Jurídicas, Universidad Internacional SEK. Quito, Ecuador. Correo electrónico: alfonso.algora@sekmail.com

*** Magíster en Seguridad y Salud Ocupacional, profesor titular de la Facultad de Ciencias del Trabajo y del Comportamiento Humano, Universidad Internacional SEK, Quito, Ecuador. Correo electrónico: marcelo.russo@ uisek.edu.ec

**** Magíster en Seguridad, Salud y Ambiente, profesor titular de la Facultad de Ciencias del Trabajo y del Comportamiento Humano, Universidad Internacional SEK, Quito, Ecuador. Correo electrónico: pablo.suasnavas@uisek.edu.ec

***** Ph. D. en Biomedicina, doctora en Medicina y Cirugía, Grupo de Investigación sobre Condiciones de Trabajo y Salud Ocupacional, Universidad Internacional SEK, Quito, Ecuador. Correo electrónico: pamela.merino@uisek. edu.ec

******* Ph. D. en Prevención de Riesgos Laborales, jefe del Grupo de Investigación sobre Condiciones de Trabajo y Salud Ocupacional, Universidad Internacional SEK, Quito, Ecuador, profesor asociado a la Facultad de Ciencias de la Salud, Universidad Internacional SEK - Chile. Correo electrónico: antonio.gomez@uisek.edu.ec 


\section{Resumen}

Introducción: los accidentes de tránsito constituyen un problema prioritario en salud pública a escala mundial, principalmente en los países de Latinoamérica, por ser una de las primeras causas de mortalidad en la región. Objetivo: estudiar la evolución y tendencia de los accidentes de tránsito en Ecuador en el periodo del 2000 al 2015. Método: estudio ecológico a partir de fuentes secundarias de información oficiales. Se presentan los resultados de las tasas de mortalidad poblacional por accidentes de tránsito y los índices de motorización, accidentabilidad, lesividad y letalidad, con el objeto de comparar las variaciones temporales en el periodo de estudio. Resultados: la tasa de mortalidad poblacional presentó una tendencia al aumento en el periodo de estudio y correlacionada con los índices de motorización y lesividad $(\mathrm{p}<0.01)$. Conclusiones: se requiere un mayor esfuerzo para optimizar las actividades destinadas al cumplimiento de las normas legales de tránsito y educación vial para la población ecuatoriana.

Palabras clave: accidentes de tránsito; mortalidad; vehículos a motor; salud pública; Ecuador

\section{Abstract}

Introduction: traffic accidents are a priority problem in public health worldwide, mainly in Latin American countries, as it is one of the leading mortality causes in the region. Objective: to study the evolution and trend of traffic accidents in Ecuador in the period from 2000 to 2015. Method: an ecological study based on secondary official information sources. The results of the population mortality rates due to traffic accidents are presented, as well as motorization, accident, harmfulness, and fatality rates, in order to compare the temporal variations in the study period. Results: the population mortality rate showed an increasing tendency in the study period and correlated with motorization and harmfulness indexes $(\mathrm{p}<0.01)$. Conclusions: a greater effort is required to optimize the activities aimed at the compliance of the legal traffic laws and towards road safety education for the Ecuadorian population.

Keywords: atraffic accidents; traffic; mortality; motor vehicles; public health; Ecuador

\section{Resumo}

Introdução: os acidentes de trânsito constituem um problema prioritário em saúde pública na escala mundial, principalmente nos países de Latino-américa, como uma das primeiras causas de mortalidade na região. Objetivo: estudar a evolução e tendência dos acidentes de trânsito no Equador no 2000 a 2015. Método: estudo ecológico a partir de fontes secundárias de informação oficiais. Apresentam-se resultados das taxas de mortalidade populacional por acidentes de trânsito e os índices de motorização, acidentalidade, lesividade e letalidade, com o objeto de comparar as variações temporais no período de estudo. Resultados: a taxa de mortalidade populacional apresentou tendência a aumentar no período de estudo e correlacionada com os índices de motorização e lesividade $(\mathrm{p}<0.01)$. Conclusões: requere-se maior esforço para otimizar as atividades destinadas ao cumprimento das regulamentações legais de trânsito e educação para a população equatoriana.

Palavras-chave: acidentes de trânsito; mortalidade; veículos a motor; saúde pública; Equador 


\section{Introducción}

Cada año fallecen, aproximadamente, 1.25 millones de personas como consecuencia de los accidentes de tránsito, y son los países de bajos ingresos los de mayor tasa de letalidad a escala mundial, lo cual constituye un problema prioritario en salud pública por el aumento de las tasas de mortalidad $(1,2)$ y los costos económicos e impactos sociales generados en los últimos años $(3,4)$. En este sentido, se estima que para el año 2030 los fallecimientos por accidentes de tránsito serán una de las principales causas de mortalidad en el mundo (5).

Según las estimaciones del informe sobre la situación mundial en seguridad vial de la Organización Mundial de la Salud, publicado en el 2015, Ecuador ocupa el séptimo puesto en Latinoamérica en tasa de fallecimientos por accidentes de tránsito y el décimo tercero a escala, global en comparación con países de ingresos similares (2). De forma similar, estudios anteriores realizados por la Escuela de Salud Pública de la Universidad de Harvard, sitúan a dichos accidentes como los responsables del $15 \%$ de las muertes en el país (6).

Diversas investigaciones recientes han empleado los estudios ecológicos de series de tiempo para conocer la problemática de estos accidentes, mediante la correlación de indicadores y tasas de mortalidad poblacional en unidades geográficas $(7,9)$.

Si se considera que los estudios citados solo proporcionan estimaciones en un periodo relativamente corto $(2,5)$ y dado que no se dispone de estudios previos en el contexto ecuatoriano, el objetivo del presente trabajo fue estudiar la evolución y la tendencia de los accidentes de tránsito en Ecuador, mediante la asociación de la tasa de mortalidad poblacional y los indicadores de motorización, accidentabilidad, lesividad y letalidad para determinar el impacto de las políticas de seguridad vial en el periodo del 2000 al 2015.

\section{Método}

Estudio ecológico sobre la evolución y tendencia de los accidentes de tránsito registrados en Ecuador entre el 2000 y el 2015, a partir de fuentes secundarias de información: censos de la población ecuatoriana y parque vehicular, a través del Instituto Nacional de Estadística y Censos (10) y la Agencia Nacional de Tránsito, como organismo encargado de registrar las estadísticas de accidentes de tránsito a escala nacional (11).

Las principales variables de estudio fueron los vehículos motorizados matriculados y el número de accidentes de tránsito, las víctimas y los fallecidos en dichos accidentes para cada año. En primer lugar, se calcularon las tasas de mortalidad poblacional por accidentes de tránsito (número de fallecidos por accidentes de tránsito x 100000 habitantes) y los índices de motorización (número de vehículos / población total), accidentabilidad (número de accidentes de tránsito / número de vehículos), lesividad (número de víctimas / número de accidentes de tránsito) y letalidad (número de fallecidos / número de víctimas) (12), con objeto de comparar las variaciones temporales en el periodo de estudio.

En segundo lugar, se calcularon las frecuencias absolutas y el porcentaje de variación interanual de los accidentes de tránsito, las víctimas y los fallecidos por dicha causa. En tercer lugar, se analizaron las tendencias temporales durante el periodo 2000-2015 de los índices de motorización, accidentabilidad, lesividad y letalidad mediante el análisis de regresión lineal simple y el cálculo del coeficiente de determinación $\left(\mathrm{R}^{2}\right)$. 
Finalmente, se estudió la correlación entre las tasas de mortalidad poblacional por accidentes de tránsito para cada uno de los índices, mediante el cálculo del coeficiente de correlación de Pearson (r), empleando a este efecto los programas estadísticos SPSS (versión 23) y Microsoft Excel 2011, este último como generador de gráficos.

\section{Resultados}

La tabla 1 muestra los valores anuales de las variables empleadas en el estudio. La población ecuatoriana se ha incrementado en 3613153 habitantes en el periodo de estudio (2000 = 12531210; $2015=16144363)$. Así mismo, se observa un aumento progresivo de los vehículos matriculados, lo cual coincide, proporcionalmente, con el número de accidentes de tránsito, víctimas y fallecidos.

La evolución de las tasas de mortalidad poblacional por accidentes de tránsito presenta un comportamiento acentuado y variado a lo largo del tiempo, principalmente en los periodos 2001-2003 y 2007-2009.

En cuanto a los indicadores objeto de estudio (figura 1.A-B-C-D), el índice de motorización presenta una tendencia lineal creciente $\left(\mathrm{R}^{2}=\right.$ 0.952), solamente afectada en el 2007 por el descenso de vehículos matriculados respecto al año anterior, a diferencia de la accidentabilidad, caracterizada por una ligera línea descendente $\left(\mathrm{R}^{2}=0.178\right)$, principalmente en el 2004 (16323) y en el 2014 (38658) por el aumento del número de accidentes de tránsito.

La lesividad, aunque en menor medida que el índice de motorización, presenta una tendencia al aumento $\left(\mathrm{R}^{2}=0.738\right)$; no obstante, se observa un ligero descenso en los últimos años del periodo analizado. Finalmente, se encuentra una tendencia a la disminución en los índices de letalidad $\left(\mathrm{R}^{2}=0.755\right)$.
Con respecto a los coeficientes de correlación de Pearson, la tasa de mortalidad por accidentes de tránsito es estadísticamente significativa de forma positiva a los índices de motorización $(\mathrm{r}=0.772 ; \mathrm{p}<0.01)$ y lesividad $(\mathrm{r}=0.886 ; \mathrm{p}<0.01)$, siendo negativa, $\mathrm{y}$ en menor medida con el índice de letalidad $(r=$ -0.675; p < 0.01). En relación con el índice de accidentabilidad, no se encontró asociación estadísticamente significativa $(\mathrm{r}=-252$; $\mathrm{p}<0.01)$ mediante el empleo del coeficiente de Spearman.

\section{Discusión}

En Ecuador se han producido 338442 accidentes de tránsito, los cuales han originado 233794 víctimas y 26811 fallecidos, datos estrechamente relacionados con el aumento del parque vehicular, el cual se incrementó en 3613153 vehículos entre el 2000 y el 2015.

De acuerdo con los resultados del presente estudio es posible llegar a varias conclusiones importantes. En primer lugar, dado que el aumento de vehículos en los países con economías emergentes es progresivo y se asocia a la tasa de mortalidad por accidentes de tránsito, es importante y necesario que los vehículos fabricados e importados en Ecuador cumplan con dispositivos básicos de seguridad activa y pasiva (2), así como el mantenimiento preventivo del vehículo por el conductor y un mayor rigor en las revisiones técnicas vehiculares por los órganos de control $(13,15)$.

En un segundo aspecto, los índices de accidentabilidad fluctúan en el periodo analizado, si bien tienden a descender ligeramente, el 2013 (28 169) y el 2014 (38658) fueron los años con mayor registro de accidentes de tránsito, con un incremento del $18 \%$ y del $37 \%$ con respecto a años anteriores. Este fenómeno se relaciona con el incremento 
del parque vehicular y, posiblemente, con el incumplimiento de las normas de tránsito por el conductor y el peatón, lo que conlleva un refuerzo y un replanteamiento de las campañas sobre educación vial en el país $(15,16)$.

En tercer lugar, los índices de lesividad presentan una tendencia al aumento, pues cada accidente de tránsito genera víctimas. Por último, en el año 2008 comienza a observarse un descenso en los índices de letalidad, salvo un ligero aumento en el año 2012. No obstante, se mantiene una línea decreciente que se estabiliza en los últimos cinco años con un promedio de 2224 fallecidos anualmente.

Si bien en este estudio no se evidencia el impacto de la normativa de tránsito y las campañas realizadas en los últimos años por la Agencia Nacional de Tránsito, el descenso del índice de accidentabilidad puede ser debido a la Ley Orgánica de Transporte Terrestre, Tránsito y Seguridad Vial y de sus posteriores modificaciones $(13,17,18)$, donde se enfatizan las medidas tendientes a prevenir o sancionar aquellas acciones que pongan en peligro el tráfico, esto último recogido en el Código Orgánico Integral Penal del 2014 que establece acciones y penas por las infracciones de tránsito, con especial atención en la responsabilidad del conductor por embriaguez o intoxicación, exceso de velocidad y pérdida de puntos en la licencia de conducir, entre otras sanciones (19).

Sin embargo, las tasas de mortalidad poblacional se han mantenido en los últimos años, las nuevas políticas no han tenido ningún efecto sobre la conducta de los ciudadanos, en particular en cuanto al cumplimiento de las normas de tránsito. El incumplimiento de la legislación a menudo socava el potencial de las leyes sobre seguridad vial para reducir la lesividad y letalidad por accidentes de tránsito, razón por la cual se requiere un mayor esfuerzo para optimizar las actividades destinadas al cumplimiento de las políticas (2).

Nuestros resultados son semejantes a otros estudios en los que se observan tendencias decrecientes de la letalidad por accidentes de tránsito, debido principalmente a la expansión y el mejoramiento en la red de carreteras y al incremento de los centros médicos hospitalarios en los últimos años (20).

Los descensos apreciados en los índices de accidentabilidad y letalidad no podrían reflejar una disminución efectiva de la problemática estudiada y enmascaran la realidad, los accidentes de tránsito siguen siendo un problema de gran preocupación para la salud pública; por ello, los resultados de este análisis pueden ser empleados para formular nuevas líneas de investigación más exhaustivas y plantear estrategias nacionales que permitan reducir la tasa de mortalidad por accidentes de tránsito (21-23).

A pesar de los objetivos planteados por la Organización Mundial de la Salud (OMS) (2), los resultados del presente estudio presentan una tendencia al aumento del índice de lesividad para el periodo de estudio, fenómeno que coincide con países de Latinoamérica como Perú (24), principalmente por el incremento desmesurado del parque vehicular en los últimos años (25).

No obstante, la tendencia a la disminución del índice de letalidad podría ser debido a la renovación del parque vehicular en los últimos años, lo cual conlleva mejoras en la seguridad activa (sistemas de frenado, dirección, suspensión, control de estabilidad, etc.) y pasiva (cinturones de seguridad, airbags, chasis-carrocería, etc.) en los nuevos vehículos. 
TABla 1. Valores y taSAS de MORTALIDAD POBlaCiOnAl POR ACCIDENTES DE TRÁNSITO EN ECUADOR: 2000-2015

\begin{tabular}{|c|c|c|c|c|c|c|c|c|c|}
\hline \multirow{2}{*}{ Año } & \multirow{2}{*}{ Población } & \multirow{2}{*}{$\begin{array}{c}\text { Vehículos } \\
\text { matriculados }\end{array}$} & \multicolumn{2}{|c|}{$\mathbf{A T}$} & \multicolumn{2}{|c|}{ Víctimas AT } & \multicolumn{2}{|c|}{ Fallecidos AT } & \multirow{2}{*}{$\begin{array}{r}\text { TM x } \\
100000\end{array}$} \\
\hline & & & $\mathrm{n}$ & Var.* (\%) & $\mathbf{n}$ & Var.* (\%) & $\mathbf{n}$ & Var.* $(\%)$ & \\
\hline 2000 & 12531210 & 290752 & 15987 & - & 7710 & - & 1099 & - & 8.8 \\
\hline 2001 & 12814503 & 594206 & 11008 & -31 & 4986 & -35 & 816 & -26 & 6.4 \\
\hline 2002 & 13093527 & 624466 & 12215 & 11 & 6357 & 27 & 1038 & 27 & 7.9 \\
\hline 2003 & 13319575 & 679584 & 10369 & -15 & 5974 & -6 & 867 & -16 & 6.5 \\
\hline 2004 & 13551875 & 726867 & 16323 & 57 & 10128 & 70 & 1273 & 47 & 9.4 \\
\hline 2005 & 13721297 & 868166 & 16578 & 2 & 10697 & 6 & 1387 & 9 & 10.1 \\
\hline 2006 & 13964606 & 962089 & 18572 & 12 & 11312 & 6 & 1801 & 30 & 12.9 \\
\hline 2007 & 14214982 & 920697 & 19598 & 6 & 13882 & 23 & 1848 & 3 & 13.0 \\
\hline 2008 & 14472881 & 989199 & 19664 & 0 & 12627 & -9 & 1343 & -27 & 9.3 \\
\hline 2009 & 14738472 & 1010465 & 21528 & 9 & 17032 & 35 & 1998 & 49 & 13.6 \\
\hline 2010 & 15012228 & 1291088 & 25588 & 19 & 20168 & 18 & 2313 & 16 & 15.4 \\
\hline 2011 & 15266431 & 1418717 & 24625 & -4 & 19113 & -5 & 2049 & -11 & 13.4 \\
\hline 2012 & 15520973 & 1509163 & 23854 & -3 & 18255 & -4 & 2242 & 9 & 14.4 \\
\hline 2013 & 15774749 & 1723975 & 28169 & 18 & 22651 & 24 & 2277 & 2 & 14.4 \\
\hline 2014 & 16027466 & 1753035 & 38658 & 37 & 27668 & 22 & 2322 & 2 & 14.5 \\
\hline 2015 & 16144363 & 1903273 & 35706 & -8 & 25234 & -9 & 2138 & -8 & 13.2 \\
\hline
\end{tabular}

* Var = variación respecto al año anterior. $\mathrm{AT}=$ accidentes de tránsito.

$\mathrm{TM}=$ tasa de fallecidos por 100000 habitantes.
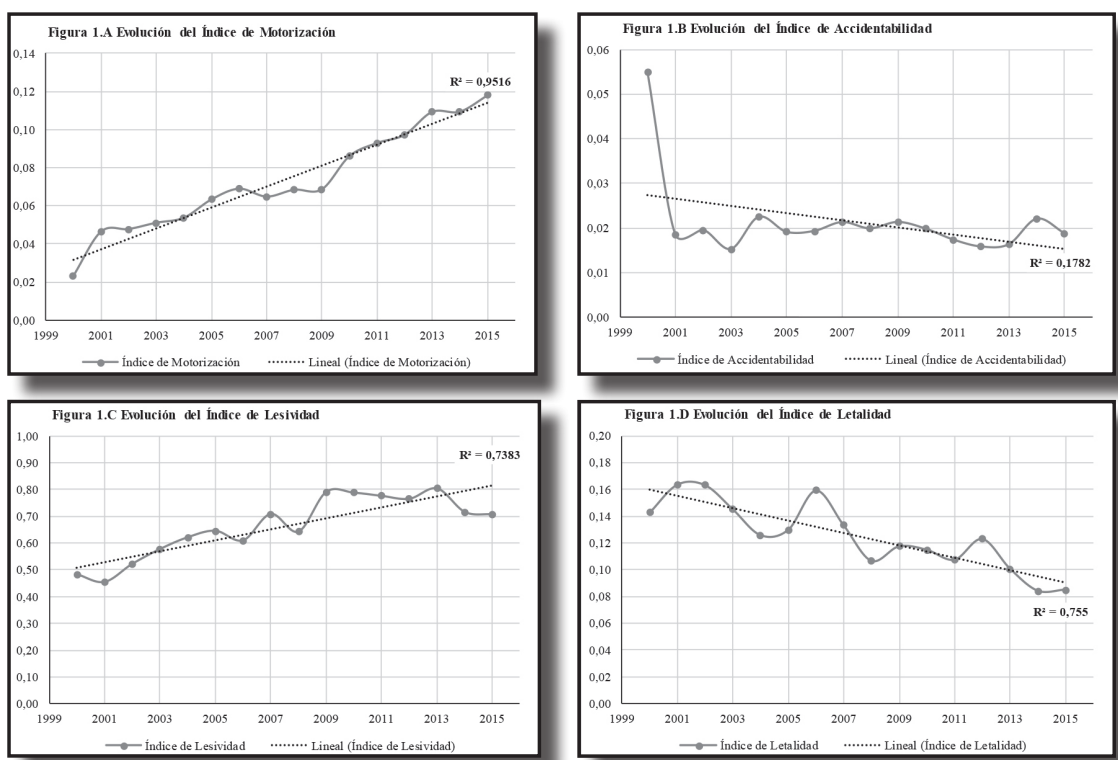

Figura 1. EVOLUCIÓN Y TENDENCIA DE LOS ÍNDICES DE MOTORIZACIÓN, ACCIDENTABILIDAD, LESIVIDAD Y LETALIDAD EN ECUADOR: 2000-2015 


\section{Referencias bibliográficas}

1. Panamerican Health Organization. Status report on road safety in Americas region. Washington, D. C.: PAHO. Disponible en: http://www.paho.org/ $\mathrm{hq} /$ index.php?option $=$ com_docman $\& \operatorname{task}=$ doc_ view\&gid $=20941 \&$ Itemid $=\overline{27} 0$

2. World Health Organization. Global status report on road safety 2015. Ginebra: WHO. Disponible en: http://apps.who.int/iris/bitstre am/10665/189242/1/9789241565066_eng.pdf?ua=1

3. Mayou R, Bryant B. Consequences of road traffic accidents for different types of road user. Injury. 2003;34:197-202.

4. Ameratunga S, Hijar M, Norton R. Road-traffic injuries: confronting disparities to address a global-health problem. Lancet.2006;367:1533-40.

5. World Health Organization. Ten statistical highlights in global public health: Part 1. Ginebra: WHO. Disponible en: http://www.who.int/whosis/ whostat2006_10highlights.pdf

6. Bhalla K, Sharaz S, Abraham J, Bartels D, Pon Hsiu Y. Road injuries in 18 countries. Departament of Global Health and Population. Harvard School of Public Health. Disponible en: http://pubdocs. worldbank.org/en/51961434469793140/Road-Safety-Injuries-18Countries-HarvardReport.pdf

7. Miranda JJ, López Rivera LA, Quistberg DA, Rosales Mayor E, Gianella C, et al. Epidemiology of road traffic incidents in Peru 1973-2008: Incidence, Mortality, and Fatality. PLoS ONE. 2014;9(6):e99662.

8. Brazinova A, Majdan M. Road traffic mortality in the Slovak Republic in 1996-2014. Traffic Inj Prev. 2016;17(7):692-8.

9. Hamad K. Road traffic accidents trends in Sharjah, United Arab Emirates during 2001-2014. International Journal of Vehicle Safety. 2016;9(1):24-38.

10. Instituto Nacional de Estadísticas y Censos. Resultados, Ecuador. Disponible en: http://www.ecuadorencifras.gob.ec//resultados

11. Agencia Nacional de Tránsito. Estadísticas de transporte terrestre y seguridad vial, Ecuador. Disponible en: http://www.ant.gob.ec/index.php/noticias/estadisticas\#.V4I6YFcWUvP

12. Sociedad Española de Epidemiología. 2007. Indicadores de morbilidad y mortalidad de lesión por accidente de tráfico. España. Disponible en: http://www.msssi. gob.es/profesionales/saludPublica/prevPromocion/ Lesiones/docs/INDICADORES.pdf
13. Agencia Nacional de Tránsito de Ecuador. Reglamento a Ley de Transporte Terrestre Transito y Seguridad Vial. Registro Oficial Suplemento N. ${ }^{\circ} 731$, 25 de junio del 2012.

14. Mirzaei M, Mirzadeh M, Shogaei Far H, Mirzaei M. Trends in road traffic deaths in Yazd, Iran, 20042010. Arch Trauma Res. 2016; 5(2):e29266.

15. Bodalal Z, Bendardaf R, Ambarek M. A Study of a decade of road traffic accidents in Benghazi-Libya: 2001 to 2010. PLoS One. 2012;7(7): e40454.

16. Bacchieri G, Barros AJ. Traffic accidents in Brazil from 1998 to 2010: many changes and few effects. Revista de Saúde Pública. 2011;45(5):949-63.

17. Agencia Nacional de Tránsito de Ecuador. Ley Orgánica de Transporte Terrestre, Tránsito y Seguridad Vial. Registro Oficial Suplemento N. ${ }^{\circ} 398$, 7 de agosto del 2008.

18. Agencia Nacional de Tránsito de Ecuador. Ley Orgánica de Transporte Terrestre, Tránsito y Seguridad Vial. Registro Oficial Suplemento N. ${ }^{\circ} 415,29$ de marzo del 2011.

19. Ecuador. Código Orgánico Integral Penal, COIP. Registro Oficial Suplemento N. ${ }^{\mathbf{0}}$ 180, 10 de febrero del 2014.

20. Xie SH, Wu YS, Liu XJ, Fu YB, Li SS, Ma HW, et al. Mortality from road traffic accidents in a rapidly urbanizing Chinese city: A 20-year analysis in Shenzhen, 1994-2013. Traffic Inj Prev. 2016;17(1):39-43.

21. Bastos JT, Shen Y, Hermans E, Brijs T, Wets G, Ferraz AC. Traffic fatality indicators in Brazil: State diagnosis based on data envelopment analysis research. Accid Anal Prev. 2015;81:61-73.

22. Wang CW, Chan CL. Estimated trends and patterns of road traffic fatalities in China, 2002-2012. Traffic Inj Prev. 2016; 17(2):164-9.

23. Restrepo Morales JA, Medina Hurtado S, Vallejo Mesa J. Pérdidas económicas para las compañías aseguradoras derivadas de personas lesionadas en accidentes de tránsito: Aplicación de un modelo de pérdidas agregadas. Rev Gerenc Polít Salud. 2016; 15(30):80-93.

24. Bambarén C, Chú M. Regulación del transporte y accidentes de tránsito por vehículos motorizados en el Perú. Rev Med Hered. 2013;24:305-10.

25. Chia L, Humani S. Accidentes de tránsito en el Perú: ¿Casualidad o causalidad? Cuadernos de Infraestructura e Inclusión Social. 2010;1(3). 